\title{
Error analysis of an explicit finite difference approximation for the space fractional diffusion equation with insulated ends
}

\author{
S. Shen* F. Liu*t \\ (Received 8 October 2004, revised 28 June 2005)
}

\begin{abstract}
The space fractional diffusion equation (SFDE) is obtained from the classical diffusion equation by replacing the second space derivative by a fractional derivative of order $\alpha, 1<\alpha \leq 2$. Numerical methods associated with integer-order differential equation, have been extensively treated. On the other hand, studies of the numerical methods and error estimates of fractional order differential equations are quite limited to date. Here, we propose an explicit finite difference approximation (EFDA) for SFDE. An error analysis of the explicit numerical method
\end{abstract}

*School of Mathematical Sciences, Xiamen University, Xiamen 361005, China. mailto:fwliu@xmu.edu.cn.

†School of Mathematical Sciences, Queensland University of Technology, Queensland 4001, Australia. mailto:f.liu@qut.edu.au.

See http://anziamj.austms.org.au/V46/CTAC2004/Shen for this article, (C) Austral. Mathematical Soc. 2005. Published September 1, 2005. ISSN 1446-8735 
for SFDE with insulated ends is discussed. We derive the scaling restriction of the stability and convergence of the explicit numerical method. Finally, some numerical results show the diffusion behaviour according to the order of space-fractional derivative and demonstrate that our EFDA is computationally simple for SFDE.

\section{Contents}

1 Introduction

C872

2 An explicit finite difference approximation for SFDE

C874

3 Method of Lines for SFDE

C875

4 Stability analysis of EFDA

C876

5 Convergence analysis of EFDA

C878

6 Numerical results

C881

7 Conclusions

C886

References

C886

\section{Introduction}

Recently, fractional derivatives have found new applications in engineering, physics, finance, and hydrology [10]. The theory of fractional calculus is a useful mathematical tool for applied sciences. Nevertheless, it is somehow hard to tackle and only in the last decades have researchers been motivated to apply the associated concepts. Podlubny [11] introduced a simple geometric 
interpretation of several types of fractional-order integration and proposed a physical interpretation of fractional integration in terms of inhomogeneous and changing (non-static, dynamic) time scale. Machado [4] presented a probabilistic interpretation of the fractional-order derivative.

Space fractional diffusion equations have been investigated by West and Seshadri [12] and more recently by Gorenflo and Mainardi [2, 3]. But numerical methods and analysis of these fractional equations are very difficult tasks. Some different numerical methods for solving the fractional partial differential equations have been proposed. Liu et al. [5, 6, 7] transformed the partial differential equation into a system of ordinary differential equations (Method of Lines), which was then solved using backward differentiation formulas. Fix and Roop [1] developed a least squares finite element solution of a fractional order two-point boundary value problem. Meerschaert et al. [8] proposed finite difference approximations for fractional advection-dispersion flow equations.

We consider the space fractional diffusion equation (SFDE) with insulated ends:

$$
\begin{aligned}
\frac{\partial u(x, t)}{\partial t} & =d(x) \frac{\partial^{\alpha} u(x, t)}{\partial x^{\alpha}}, \quad 0<x<L, \quad t \geq 0, \quad 1<\alpha \leq 2, \\
u(x, 0) & =\psi(x), \quad 0 \leq x \leq L, \\
\frac{\partial u(0, t)}{\partial x} & =0, \quad \frac{\partial u(L, t)}{\partial x}=0, \quad t \geq 0,
\end{aligned}
$$

where variable coefficient $d(x)>0, \partial^{\alpha} u(x, t) / \partial x^{\alpha}$ is Caputo's fractional derivative ${ }_{0} D_{x}^{\alpha} u(x)$, which is defined as [10]

$$
\frac{\partial^{\alpha} u}{\partial x^{\alpha}}={ }_{0} D_{x}^{\alpha} u(x)= \begin{cases}\frac{d^{m} u(x)}{d x^{m}}, & \alpha=m \in N \\ \frac{1}{\Gamma(m-\alpha)} \int_{0}^{x}(x-\xi)^{m-\alpha-1} \frac{d^{m} u(\xi)}{d \xi^{m}} d \xi, & m-1<\alpha<m\end{cases}
$$

where $\Gamma(\cdot)$ is the gamma function.

In this paper, an explicit finite difference approximation for the SFDE is presented. The stability and convergence of the explicit finite difference 
approximation are analyzed, respectively. Finally, some results show that our numerical method is computationally simple and efficient.

\section{An explicit finite difference approximation for SFDE}

Suppose $h=x / k, k$ is a positive integer. Using a second order difference approximation, we get

$$
\begin{aligned}
& { }_{0} D_{x}^{\alpha} u(x, t) \\
& =\frac{1}{\Gamma(2-\alpha)} \int_{0}^{x} \frac{1}{(x-\xi)^{\alpha-1}} \frac{\partial^{2} u(\xi, t)}{\partial \xi^{2}} d \xi \\
& =\frac{1}{\Gamma(2-\alpha)} \sum_{j=0}^{k-1} \int_{j h}^{(j+1) h} z^{1-\alpha} \frac{\partial^{2} u(x-z, t)}{\partial z^{2}} d z \\
& \approx \frac{1}{\Gamma(2-\alpha)} \sum_{j=0}^{k-1} \frac{u(x-(j-1) h, t)-2 u(x-j h, t)+u(x-(j+1) h, t)}{h^{2}} \\
& \quad \times \int_{j h}^{(j+1) h} z^{1-\alpha} d z \\
& =\frac{h^{-\alpha}}{\Gamma(3-\alpha)} \sum_{j=0}^{k-1}[u(x-(j-1) h, t)-2 u(x-j h, t)+u(x-(j+1) h, t)] \\
& \quad \times\left[(j+1)^{2-\alpha}-j^{2-\alpha}\right] .
\end{aligned}
$$

Let $\Delta t=\tau>0$ be the grid step in time, $t_{n}=n \tau, 0 \leq t_{n} \leq T, \Delta x=$ $h>0$ be the grid step in space, $x_{j}=j h, 0 \leq x_{j} \leq L$ for $j=0,1, \ldots, K$, $K=L / h$. Let $u_{0}^{n}=u(0, n \tau), u_{1}^{n}=u(h, n \tau), \ldots, u_{k-j}^{n}=u((k-j) h, n \tau)$, $\ldots, u_{j}^{n}=u(j h, n \tau) ; d_{j}=d\left(x_{j}\right) ; \psi_{j}=\psi\left(x_{j}\right)$. 
Now we approximate SFDE (1) by using an explicit finite-difference approximation (EFDA):

$$
\frac{u_{k}^{n+1}-u_{k}^{n}}{\tau}=\frac{d_{k} h^{-\alpha}}{\Gamma(3-\alpha)} \sum_{j=0}^{k-1}\left[u_{k-j+1}^{n}-2 u_{k-j}^{n}+u_{k-j-1}^{n}\right]\left[(j+1)^{2-\alpha}-j^{2-\alpha}\right] .
$$

Equation (6) can be rewritten as

$$
u_{k}^{n+1}=b_{k} u_{k+1}^{n}+\left(1-2 b_{k}\right) u_{k}^{n}+b_{k} u_{k-1}^{n}+b_{k} \sum_{j=1}^{k-1} g_{j}\left[u_{k-j+1}^{n}-2 u_{k-j}^{n}+u_{k-j-1}^{n}\right] \text {, }
$$

where $b_{k}=\tau d_{k} /\left[h^{\alpha} \Gamma(3-\alpha)\right], g_{k}=(k+1)^{2-\alpha}-k^{2-\alpha}$.

Equation (7), together with the boundary conditions $\left(u_{0}^{n}=u_{1}^{n}, u_{K-1}^{n}=\right.$ $\left.u_{K}^{n}\right)$, result in the following linear system of equations:

$$
U^{n+1}=A U^{n},
$$

where $U^{n}=\left(u_{1}^{n}, u_{2}^{n}, \ldots, u_{K-1}^{n}\right)^{T}$, and $A=\left(a_{i j}\right)$ is a matrix of coefficients. These coefficients, for $i=1,2, \ldots, K-1$ and $j=2,3, \ldots, K-1$ are

$$
a_{i j}= \begin{cases}0, & \text { when } j \geq i+2, \\ b_{i}, & \text { when } j=i+1, \\ 1-b_{i}\left(2-g_{1}\right), & \text { when } j=i=2,3, \ldots, K-2, \\ b_{i}\left(1-2 g_{1}+g_{2}\right), & \text { when } j=i-1, \\ b_{i}\left(g_{i-j-1}-2 g_{i-j}+g_{i-j+1}\right), & \text { when } j \leq i-2,\end{cases}
$$

while $a_{11}=1-b_{1}, a_{21}=b_{2}\left(1-g_{1}\right), a_{i 1}=b_{i}\left(g_{i-2}-g_{i-1}\right)$, for $3 \leq i \leq K-1$, $a_{K-1, K-1}=1-b_{K-1}\left(1-g_{1}\right)$.

\section{Method of Lines for SFDE}

In order to demonstrate the simplicity and efficiency of the EFDA, the method of lines for SFDE also is presented. This method of lines (MOL) is firstly 
introduced by Liu et al. $[5,6,7]$ and has been used to solve fractional partial differential equations successfully. The method of lines for SFDE can be written as the following form: for $1<\alpha<2,(k=1, \ldots, K-1)$,

$$
\frac{d u_{k}}{d t}=\bar{b}_{k} \sum_{j=0}^{k-1} g_{j}\left[u_{k-j+1}-2 u_{k-j}+u_{k-j-1}\right]
$$

with $\bar{b}_{k}=b_{k} / \tau, u_{0}=u_{1}, u_{K}=u_{K-1}$ and $u_{j}=u\left(x_{j}, t\right)$.

\section{Stability analysis of EFDA}

Lemma 1 Let $A \in C^{n \times n}$ and $\rho(A)$ is the spectral radius of the matrix $\mathrm{A}$, then for any given positive number $\varepsilon$, there exists a norm $\|\cdot\|_{m}$ of the matrix A such that $\|A\|_{m} \leq \rho(A)+\varepsilon$.

Proof: See [13].

Theorem 2 The explicit finite-difference scheme (6) for SFDE (1)-(3) is conditionally stable.

Proof: Let $\lambda$ be an eigenvalue of the matrix $A$ to linear system of equations (8), so that $A x=\lambda x$ for some nonzero vector $x$. Choose $i$ so that $\left|x_{i}\right|=\max \left\{\left|x_{j}\right|: j=1,2, \ldots, K-1\right\}$, then $\sum_{j=1}^{K-1} a_{i j} x_{j}=\lambda x_{i}$, and therefore

$$
\lambda=a_{i i}+\sum_{j=1, j \neq i}^{K-1} a_{i j} \frac{x_{j}}{x_{i}} .
$$

Substituting the values of $a_{i j}$ into (11) we get 
1. when $i=1$ :

$$
\lambda=1-b_{1}+b_{1} \frac{x_{2}}{x_{1}} \leq 1
$$

and

$$
\lambda=1-b_{1}+b_{1} \frac{x_{2}}{x_{1}} \geq 1-2 b_{1} .
$$

If $b_{1} \leq 1$, we have $|\lambda| \leq 1$.

2. when $2 \leq i \leq K-2$ :

$$
\begin{aligned}
\lambda= & 1-b_{i}\left(2-g_{1}\right)+b_{i} \frac{x_{i+1}}{x_{i}}+b_{i} \sum_{j=2}^{i-1}\left(g_{i-j-1}-2 g_{i-j}+g_{i-j+1}\right) \frac{x_{j}}{x_{i}} \\
& +b_{i}\left(g_{i-2}-g_{i-1}\right) \frac{x_{1}}{x_{i}}
\end{aligned}
$$

We note that $g_{i}>g_{i+1}>0, g_{i-j-1}-2 g_{i-j}+g_{i-j+1}>0$, for $j=$ $1,2, \ldots, i-1, i=0,1, \ldots, K-1$, we have

$$
\sum_{j=2}^{i-1}\left(g_{i-j-1}-2 g_{i-j}+g_{i-j+1}\right) \frac{x_{j}}{x_{i}} \leq g_{i-1}-g_{i-2}+g_{0}-g_{1} .
$$

Since $b_{i}$ are non-negative real numbers, from Equation (12), we can get $\lambda \leq 1-b_{i}\left(2-g_{1}\right)+b_{i}+b_{i}\left(g_{i-1}-g_{i-2}+g_{0}-g_{1}\right)+b_{i}\left(g_{i-2}-g_{i-1}\right)=1$ and

$$
\begin{aligned}
\lambda & \geq 1-b_{i}\left(2-g_{1}\right)-b_{i}-b_{i}\left(g_{i-1}-g_{i-2}+g_{0}-g_{1}\right)-b_{i}\left(g_{i-2}-g_{i-1}\right) \\
& =1-2 b_{i}\left(2-g_{1}\right) .
\end{aligned}
$$

If $b_{i}\left(2-g_{1}\right) \leq 1$, then $\lambda \geq-1$. Hence $|\lambda| \leq 1$.

3. when $i=K-1$ :

$$
\lambda=1-b_{K-1}\left(1-g_{1}\right)+b_{K-1} \sum_{j=2}^{i-1}\left(g_{i-j-1}-2 g_{i-j}+g_{i-j+1}\right) \frac{x_{j}}{x_{i}}
$$




$$
+b_{K-1}\left(g_{i-2}-g_{i-1}\right) \frac{x_{1}}{x_{i}}
$$

Thus

$$
\begin{aligned}
\lambda \leq & 1-b_{K-1}\left(1-g_{1}\right)+b_{K-1}\left(g_{i-1}-g_{i-2}+g_{0}-g_{1}\right) \\
& +b_{K-1}\left(g_{i-2}-g_{i-1}\right) \\
= & 1
\end{aligned}
$$

and

$$
\begin{aligned}
\lambda \geq & 1-b_{K-1}\left(1-g_{1}\right)-b_{K-1}\left(g_{i-1}-g_{i-2}+g_{0}-g_{1}\right) \\
& -b_{K-1}\left(g_{i-2}-g_{i-1}\right) \\
= & 1-2 b_{K-1}\left(1-g_{1}\right) .
\end{aligned}
$$

If $b_{K-1}\left(1-g_{1}\right) \leq 1$, then $\lambda \geq-1$. Hence $|\lambda| \leq 1$.

Combining 1, 2 and 3 , we have that if $\max _{2 \leq i \leq K-2}\left\{b_{1}, b_{i}\left(2-g_{1}\right), b_{K-1}(1-\right.$ $\left.\left.g_{1}\right)\right\} \leq 1$, the spectral radius $\rho(A)$ of the matrix satisfies $\rho(A) \leq 1$. From Lemma 1 , we get that if $\max _{2 \leq i \leq K-2}\left\{b_{1}, b_{i}\left(2-g_{1}\right), b_{K-1}\left(1-g_{1}\right)\right\} \leq 1$, there exists a positive number $\varepsilon \leq C \tau$ such that $\|A\|_{m} \leq \rho(A)+C \tau \leq 1+\mathcal{O}(\tau)$. Therefore, EFDA (6) is conditionally stable.

\section{Convergence analysis of EFDA}

Lemma 3 Let

$$
\overline{{ }_{0} D_{x}^{\alpha} u(x, t)}=\frac{h^{-\alpha}}{\Gamma(3-\alpha)} \sum_{j=0}^{k-1} g_{j}\left[u_{k-j+1}^{n}-2 u_{k-j}^{n}+u_{k-j-1}^{n}\right]
$$

be a smooth function, then

$$
{ }_{0} D_{x}^{\alpha} u(x, t)=\overline{{ }_{0} D_{x}^{\alpha} u(x, t)}+\mathcal{O}(h) .
$$


Proof: In term of standard centered difference formula, we have

$$
\begin{aligned}
\overline{{ }_{0} D_{x}^{\alpha} u(x, t)} & =\frac{h^{2-\alpha}}{\Gamma(3-\alpha)} \sum_{j=0}^{k-1} g_{j}\left[\frac{\partial^{2} u(x-j h, t)}{\partial z^{2}}+\mathcal{O}\left(h^{2}\right)\right] \\
& =\frac{h^{2-\alpha}}{\Gamma(3-\alpha)} \sum_{j=0}^{k-1} g_{j} \frac{\partial^{2} u(x-j h, t)}{\partial z^{2}}+\frac{h^{2-\alpha} k^{2-\alpha}}{\Gamma(3-\alpha)} \mathcal{O}\left(h^{2}\right) \\
& =\frac{h^{2-\alpha}}{\Gamma(3-\alpha)} \sum_{j=0}^{k-1} g_{j} \frac{\partial^{2} u(x-j h, t)}{\partial z^{2}}+\frac{x^{2-\alpha}}{\Gamma(3-\alpha)} \mathcal{O}\left(h^{2}\right) \\
& =\frac{h^{2-\alpha}}{\Gamma(3-\alpha)} \sum_{j=0}^{k-1} g_{j} \frac{\partial^{2} u(x-j h, t)}{\partial z^{2}}+\mathcal{O}\left(h^{2}\right) .
\end{aligned}
$$

By the integral mean value theorem, we have

$$
\begin{aligned}
{ }_{0} D_{x}^{\alpha} u(x, t) & =\frac{1}{\Gamma(2-\alpha)} \sum_{j=0}^{k-1} \int_{j h}^{(j+1) h} z^{1-\alpha} \frac{\partial^{2} u(x-z, t)}{\partial z^{2}} d z \\
& =\frac{h^{2-\alpha}}{\Gamma(3-\alpha)} \sum_{j=0}^{k-1} g_{j} \frac{\partial^{2} u\left(x-\xi_{j}, t\right)}{\partial z^{2}}
\end{aligned}
$$

where $\xi_{j} \in[j h,(j+1) h]$. Combining the above two formulae, we have

$$
\begin{aligned}
& \left|\overline{{ }_{0} D_{x}^{\alpha} u(x, t)}-{ }_{0} D_{x}^{\alpha} u(x, t)\right| \\
& \quad=\left|\frac{h^{2-\alpha}}{\Gamma(3-\alpha)} \sum_{j=0}^{k-1} g_{j}\left[\frac{\partial^{2} u(x-j h, t)}{\partial z^{2}}-\frac{\partial^{2} u\left(x-\xi_{j}, t\right)}{\partial z^{2}}\right]+\mathcal{O}\left(h^{2}\right)\right| \\
& \quad=\left|\frac{h^{2-\alpha}}{\Gamma(3-\alpha)} \sum_{j=0}^{k-1} g_{j} \cdot \mathcal{O}(h)+\mathcal{O}\left(h^{2}\right)\right| \\
& \quad=\left|\frac{h^{2-\alpha} k^{2-\alpha}}{\Gamma(3-\alpha)} \cdot \mathcal{O}(h)+\mathcal{O}\left(h^{2}\right)\right|
\end{aligned}
$$




$$
\begin{aligned}
& =\mathcal{O}(h)+\mathcal{O}\left(h^{2}\right) \\
& =\mathcal{O}(h) .
\end{aligned}
$$

Remark 4 The explicit finite-difference scheme (6) has a local truncation error of $e_{r}=\mathcal{O}(\tau+h)$.

Theorem 5 if $\max _{2 \leq i \leq K-2}\left\{b_{1}, b_{i}\left(2-g_{1}\right), b_{K-1}\left(1-g_{1}\right)\right\} \leq 1$, then the explicit finite-difference scheme (6) for SFDE (1)-(3) is convergent, and the order of convergence is $\mathcal{O}(\tau+h)$.

Proof: At the mesh points $\left(x_{k}, t_{n}\right), y_{k}^{n}=u_{k}^{n}-e_{k}^{n}$. Substitution into (6) leads to

$$
\begin{aligned}
\frac{\left(u_{k}^{n+1}-e_{k}^{n+1}\right)-\left(u_{k}^{n}-e_{k}^{n}\right)}{\tau}= & \frac{d_{k} h^{-\alpha}}{\Gamma(3-\alpha)} \sum_{j=0}^{k-1} g_{j}\left[\left(u_{k-j+1}^{n}-2 u_{k-j}^{n}+u_{k-j-1}^{n}\right)\right. \\
& \left.-\left(e_{k-j+1}^{n}-2 e_{k-j}^{n}+e_{k-j+1}^{n}\right)\right] .
\end{aligned}
$$

Using the Taylor theorem and Lemma 3, we obtain

$$
\begin{aligned}
& {\left[\frac{\partial u}{\partial t}\right]_{k}^{n}+\mathcal{O}(\tau)-\frac{e_{k}^{n+1}-e_{k}^{n}}{\tau}} \\
& \quad=d_{k}\left[\frac{\partial^{\alpha} u}{\partial x^{\alpha}}+\mathcal{O}(h)\right]-\frac{d_{k} h^{-\alpha}}{\Gamma(3-\alpha)} \sum_{j=0}^{k-1} g_{j}\left(e_{k-j+1}^{n}-2 e_{k-j}^{n}+e_{k-j-1}^{n}\right) .
\end{aligned}
$$

Thus, we have

$$
\frac{e_{k}^{n+1}-e_{k}^{n}}{\tau}=\frac{d_{k} h^{-\alpha}}{\Gamma(3-\alpha)} \sum_{j=0}^{k-1} g_{j}\left(e_{k-j+1}^{n}-2 e_{k-j}^{n}+e_{k-j-1}^{n}\right)+[\mathcal{O}(\tau+h)] .
$$


Using the initial and boundary conditions $e_{k}^{0}=0, e_{0}^{n+1}=e_{1}^{n+1}, e_{K-1}^{n+1}=e_{K}^{n+1}$, Equation (16) can be rewritten in matrix form:

$$
E_{n+1}=A E_{n}+M, \quad E_{0}=0,
$$

where $E_{n}=\left(e_{1}^{n}, e_{2}^{n}, \ldots, e_{K-1}^{n}\right)^{T}$ and $M=\tau(\mathcal{O}(\tau+h))(1,1, \ldots, 1)^{T}$. Hence we can get

$$
E_{n+1}=\left(A^{n}+A^{n-1}+\cdots+A^{2}+A+I\right) M .
$$

Thus

$$
\left\|E_{n+1}\right\|_{\infty} \leq\left(\left\|A^{n}\right\|_{\infty}+\left\|A^{n-1}\right\|_{\infty}+\cdots+\|A\|_{\infty}+\|I\|_{\infty}\right)\|M\|_{\infty} .
$$

Also

$$
\begin{aligned}
\|A\|_{\infty}= & \max _{1 \leq i \leq K-1} \sum_{j=1}^{K-1}\left|a_{i j}\right| \\
= & \max \left\{\left|1-b_{1}\right|+b_{1}, \max _{2 \leq i \leq K-1}\left[\left|1-b_{i}\left(2-g_{1}\right)\right|+b_{i}\left(2-g_{1}\right)\right],\right. \\
& \left.\left|1-b_{K-1}\left(1-g_{1}\right)\right|+b_{K-1}\left(1-g_{1}\right)\right\} .
\end{aligned}
$$

If $\max _{2 \leq i \leq K-2}\left\{b_{1}, b_{i}\left(2-g_{1}\right), b_{K-1}\left(1-g_{1}\right)\right\} \leq 1$, then $\|A\|_{\infty} \leq 1$. Thus we can get

$$
\left\|E_{n+1}\right\|_{\infty} \leq(n+1) \tau|\mathcal{O}(\tau+h)| .
$$

Consequently, when $\tau \rightarrow 0, h \rightarrow 0$, we have $\left|e_{k}^{n+1}\right| \rightarrow 0$. This proves that $y$ converges to $u$ as $\tau$ and $h$ tend to zero if $\max _{2 \leq i \leq K-2}\left\{b_{1}, b_{i}\left(2-g_{1}\right), b_{K-1}(1-\right.$ $\left.\left.g_{1}\right)\right\} \leq 1$.

\section{$6 \quad$ Numerical results}

In this section, the following space fractional diffusion equation (SFDE) with insulated ends is considered:

$$
\frac{\partial u(x, t)}{\partial t}=d \frac{\partial^{\alpha} u(x, t)}{\partial x^{\alpha}}, \quad 0<x<\pi, \quad t \geq 0, \quad 1<\alpha \leq 2,
$$




$$
\begin{aligned}
u(x, 0) & =\psi(x)=x^{2}, \quad 0 \leq x \leq \pi, \\
\frac{\partial u(0, t)}{\partial x} & =0, \quad \frac{\partial u(\pi, t)}{\partial x}=0, \quad t \geq 0 .
\end{aligned}
$$

When $\alpha=2, d$ is a constant, the analytical solution of the heat equation with insulated ends [9] is

$$
u(x, t)=\frac{1}{3} \pi^{2}+\sum_{n=1}^{\infty} \frac{4(-1)^{n}}{n^{2}} \cos n x e^{-n^{2} d t} .
$$

EFDA is an explicit method. MoL is a computationally efficient method [6, 7]. In Figure 1(a), the analytical solution, numerical solutions (MOL) and EFDA for $\alpha=2, d=0.4, t=0.3$ are shown. See in Figure 1(a) that both numerical solutions are in good agreement with the analytical solution. In Figure 1(b), the numerical solutions using MoL and EFDA with $h=\pi / 100$, $\tau=0.0001$ for $\alpha=1.7, d=0.4, t=0.3$ are shown. From Figure $1(\mathrm{~b})$, see that EFDA is in good agreement with MoL. It demonstrates that our EFDA is an computationally simple and efficient method for SFDE.

Tables 1 and 2 compare MoL and EFDA solutions with $h=\pi / 100, \tau=$ $0.0001, \alpha=1.7$ at $t=0.3$ and $t=1.0$, respectively. From Tables 1 and 2 , see that the solution from EFDA agrees well with MoL solution, and hints at a convergence order of at least one.

Figure 2(a) shows the evolution results using EFDA with $h=\pi / 100$, $\tau=0.0001, \alpha=1.7(0 \leq t \leq 1,0 \leq x \leq \pi)$. Figure 2(b) shows the response of the diffusion system at $t=0.3$ using EFDA for different real numbers $1.5 \leq \alpha \leq 2$. These numerical results show the diffusion behaviour according to the order of space-fractional derivative. From Figure 2, see that this method applies to solve fractional order differential equations. 


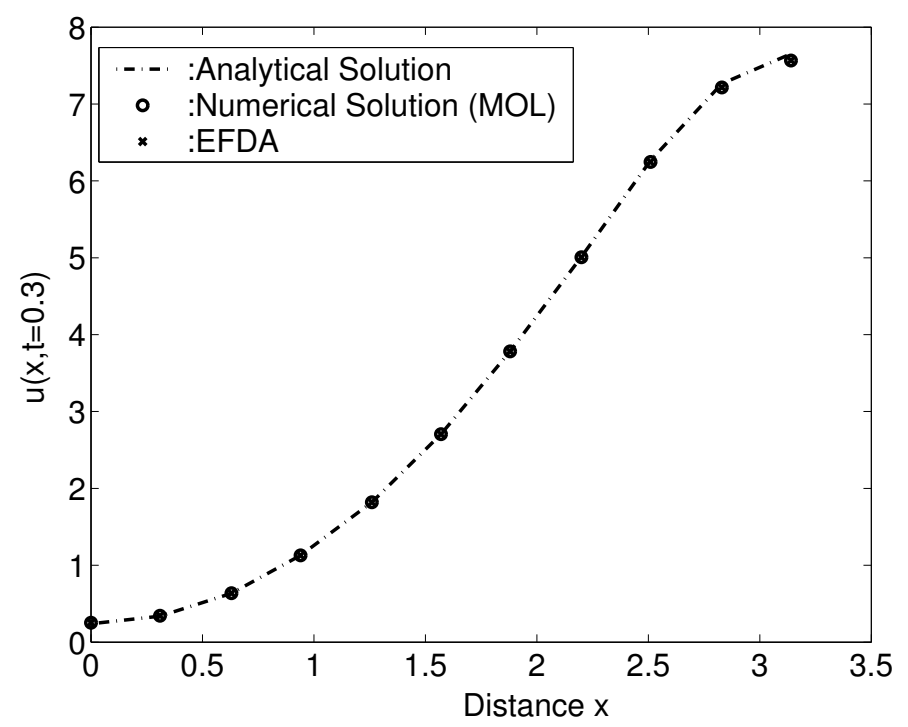

(a)

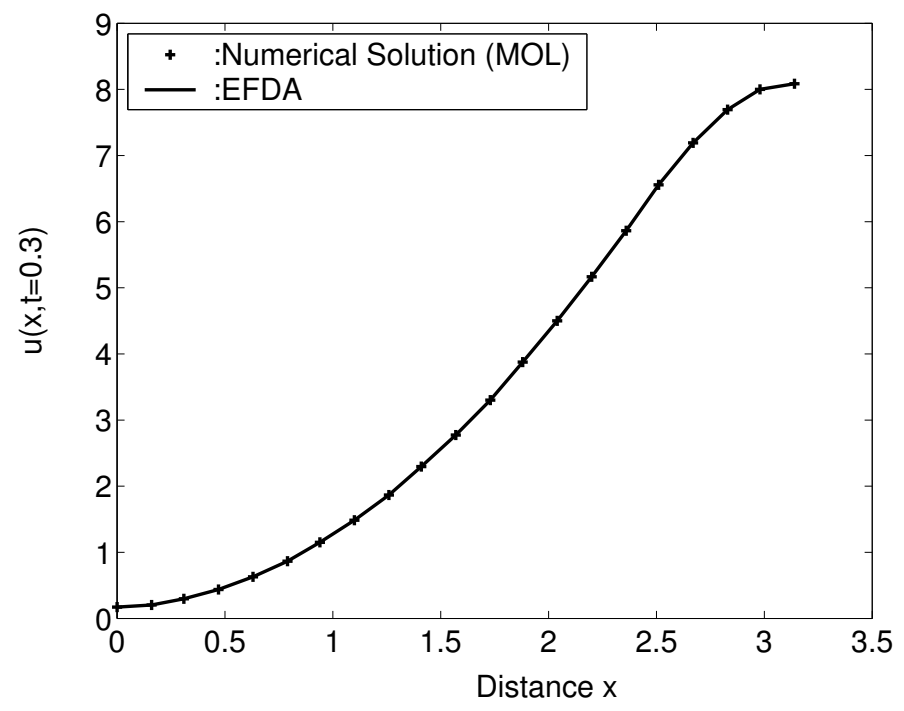

Figure 1: (a) Comparison of the analytical, MoL and EFDA with $\alpha=2$, $d=0.4, t=0.3$; (b) Comparison of MoL and EFDA with $h=\pi / 100$, $\tau=0.0001, \alpha=1.7$. 
TABLE 1: Comparison of MoL and EFDA with $h=\pi / 100, \tau=0.0001$, $\alpha=1.7, t=0.3$.

\begin{tabular}{|c|ccc|}
\hline$x_{i}$ & MOL & EFDA & MOL-EFDA \\
\hline 0.0000 & 0.17059802 & 0.17059147 & $0.654161 \mathrm{E}-05$ \\
0.3142 & 0.29611441 & 0.29611009 & $0.432134 \mathrm{E}-05$ \\
0.6283 & 0.62850708 & 0.62850535 & $0.172853 \mathrm{E}-05$ \\
0.9425 & 1.15109479 & 1.15109420 & $0.596046 \mathrm{E}-06$ \\
1.2566 & 1.86552680 & 1.86552656 & $0.238419 \mathrm{E}-06$ \\
1.5708 & 2.77362752 & 2.77362752 & $0.000000 \mathrm{E}+00$ \\
1.8850 & 3.87621999 & 3.87622404 & $-.405312 \mathrm{E}-05$ \\
2.1991 & 5.16582966 & 5.16587639 & $-.467300 \mathrm{E}-04$ \\
2.5133 & 6.55614185 & 6.55631781 & $-.175953 \mathrm{E}-03$ \\
2.8274 & 7.69319010 & 7.69348478 & $-.294685 \mathrm{E}-03$ \\
3.1416 & 8.08523273 & 8.08554745 & $-.314713 \mathrm{E}-03$ \\
\hline
\end{tabular}

TABLE 2: Comparison of MoL and EFDA with $h=\pi / 100, \tau=0.0001$, $\alpha=1.7, t=1.0$.

\begin{tabular}{|c|ccc|}
\hline$x_{i}$ & MOL & EFDA & MOL-EFDA \\
\hline 0.0000 & 0.66449213 & 0.66448492 & $0.721216 \mathrm{E}-05$ \\
0.3142 & 0.81328374 & 0.81327778 & $0.596046 \mathrm{E}-05$ \\
0.6283 & 1.19325054 & 1.19324791 & $0.262260 \mathrm{E}-05$ \\
0.9425 & 1.76574183 & 1.76574767 & $-.584126 \mathrm{E}-05$ \\
1.2566 & 2.51690125 & 2.51692772 & $-.264645 \mathrm{E}-04$ \\
1.5708 & 3.42062283 & 3.42068386 & $-.610352 \mathrm{E}-04$ \\
1.8850 & 4.41448212 & 4.41458607 & $-.103951 \mathrm{E}-03$ \\
2.1991 & 5.38522577 & 5.38537598 & $-.150204 \mathrm{E}-03$ \\
2.5133 & 6.18573856 & 6.18592167 & $-.183105 \mathrm{E}-03$ \\
2.8274 & 6.68846416 & 6.68865585 & $-.191689 \mathrm{E}-03$ \\
3.1416 & 6.84125662 & 6.84144831 & $-.191689 \mathrm{E}-03$ \\
\hline
\end{tabular}




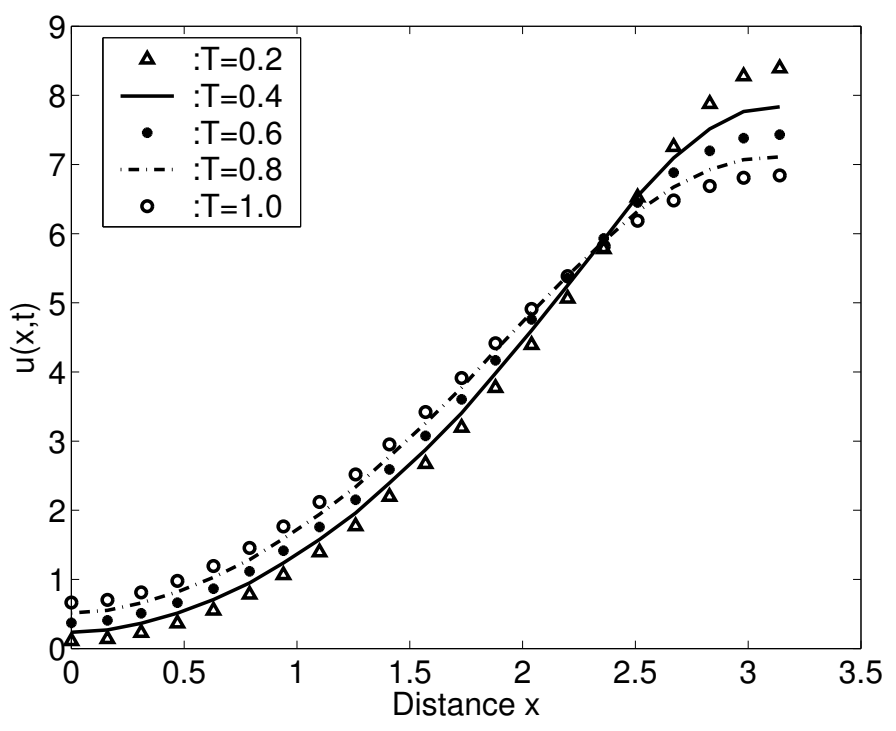

(a)

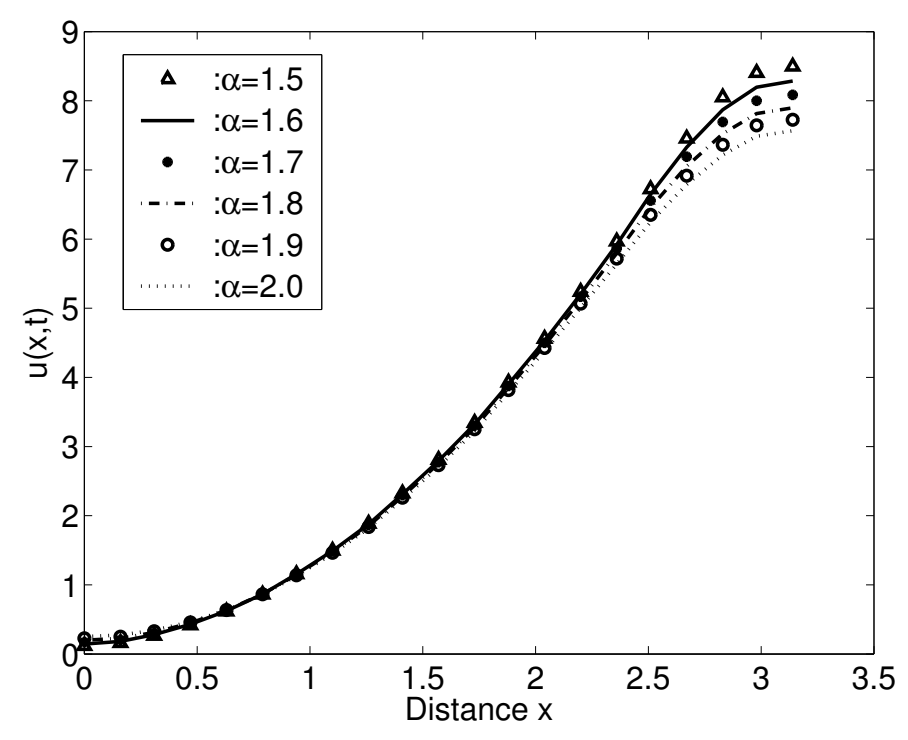

(b)

Figure 2: (a) The evolution results using EFDA with $h=\pi / 100, \tau=$ $0.0001, \alpha=1.7(0 \leq t \leq 1,0 \leq x \leq \pi)$; (b) Comparison of the response of the diffusion system using EFDA for different real numbers $1.5 \leq \alpha \leq 2$. 


\section{Conclusions}

An explicit finite difference approximation (EFDA) and Method of Lines (MOL) for SFDE are explored. Error analysis, stability and convergence of the explicit numerical method for SFDE with insulated ends are discussed. Finally, some numerical results of EFDA and MoL are presented. These numerical results demonstrate that our EFDA is a computationally simple and efficient method for SFDE. This method can be applied to solve fractional differential equations.

Acknowledgment: This research has been supported by the National Natural Science Foundation of China grant 10271098.

\section{References}

[1] G. J. Fix and J. P. Roop, Least squares finite element solution of a fractional order two-point boundary value problem, Comput. Math. Appl., 48, 2004, 1017-1044. C873

[2] R. Gorenflo and F. Mainardi, Random walk models for space fractional diffusion processes, Fractional Calculus \& Applied Analysis 1, 1998, 167-191. C873

[3] R. Gorenflo and F. Mainardi, Approximation of Levy-Feller diffusion by random walk, Journal for Analysis and its Applications (ZAA), 18, 1999, 231-246. C873

[4] J. A. Tenreiro Machado, A probabilistic interpretation of the fractional-order differentiation, Fractional Calculus \& Applied Analysis, 6, No.1, 2003, 73-80. C873 
[5] F. Liu, V. Anh and I. Turner, Numerical solution of the fractional-order Advection-Dispersion Equation, The Procceding of An International Conference on Boundary and Interior Layers-Computational and Asymptotic Methods, Perth, Australia, 2002, 159-164. C873, C876

[6] F. Liu, V. Anh, I. Turner, Numerical solution of the space fractional Fokker-Planck equation, Journal of Computational and Applied Mathematics 166, 2004, 209-219. C873, C876, C882

[7] F. Liu, V. Anh, I. Turner and P. Zhuang, Numerical simulation for solute transport in fractal porous media, ANZIAM J., 45(E), 2004, C461-C473. C873, C876, C882

[8] M. M. Meerschaert and C. Tadjeran, Finite difference approximations for fractional advection-dispersion flow equations, Journal of Computational and Applied Mathematics, 172, 2004, 65-77. C873

[9] P. V. O'Neil, Advanced Engineering Mathematics, Thomson Brooks/Cole, 2003. C882

[10] I. Podlubny, Fractional Differential Equations, Academic, Press, New York, 1999. C872, C873

[11] I. Podlubny, Geometric and Physical Interpretation of Fractional Integration and Fractional Differentiation, Fractional Calculus 83 Applied Analysis, 5, No.4, 2002, 367-386. C872

[12] B. West and V. Seshadri, Linear systems with Levy fluctuations, Physica A, 113, 1982, 203-216. C873

[13] Z. Xu, K. Zhang, Q. Lu, G. Leng, Matrix Theory, Scientific publishing house, 2001. C876 